VoL. 60 (1999) [483-486]

\title{
ON ISOCOMPACTNESS OF FUNCTION SPACES
}

\author{
JiLING CAO
}

Let $C_{p}(X)$ be the space of all continuous real-valued functions on a Tychonoff space $X$ with the pointwise topology. In this note, we show that if $X$ is a $\mathcal{G}$-space, then $C_{p}(X)$ is isocompact. This gives an answer to a recent question of Arkhangel'skii in the class of $\mathcal{G}$-spaces.

\section{INTRODUCTION}

In studying the compactness of countably compact spaces, Bacon [3] introduced the notion of an isocompact space. Recall that a topological space $X$ is isocompact, if every closed countably compact subspace of $X$ is compact. Obviously, any topological property, which makes a countably compact space compact, implies isocompactness. Among the classes of spaces which are isocompact are the $\theta$-refinable spaces [10], the spaces having a $G_{\delta}$-diagonal [5], and the symmetrisable spaces [9], to name a few. The main purpose of this note is to study the isocompactness of function spaces and to answer a recent question of Arkhangel'skii in the class of $\mathcal{G}$-spaces defined by a two-person game.

For a Tychonoff space $X$, let

$$
C(X)=\{f: X \rightarrow \mathbf{R} \text { is continuous }\}
$$

that is, $C(X)$ is the family of all continuous real-valued functions defined on $X$. We shall denote by $C_{p}(X)$ the space of $C(X)$, endowed with the topology of pointwise convergence on $X$. Obviously, a basic neighbourhood of a function $f \in C_{p}(X)$ is

$$
W\left(x_{0}, x_{1}, \ldots, x_{n} ; \varepsilon\right)(f)=\left\{g \in C(X):\left|f\left(x_{i}\right)-g\left(x_{i}\right)\right|<\varepsilon, 0 \leqslant i \leqslant n\right\}
$$

where $\varepsilon>0$ and $x_{0}, x_{1}, \ldots, x_{n} \in X$.

No separation axioms are assumed on topological spaces if it is not stated explicitly, and more information on $C_{p}$-theory can be found in [1].

Received 8th March, 1999

Copyright Clearance Centre, Inc. Serial-fee code: $0004-9727 / 99 \$ A 2.00+0.00$. 


\section{MAIN THEOREM}

Let $X$ be a topological space, and let $x \in X$ be a point. The family of all neighbourhoods of $x$ is denoted by $\mathcal{U}(x)$. We shall consider the following $\mathcal{G}(x)$-game [4] played in $X$ between two players $(\alpha)$ and $(\beta)$. Player $(\alpha)$ goes first and chooses a point $x_{1} \in X$. Player $(\beta)$ then responds by choosing $U_{1} \in \mathcal{U}(x)$. Following this, player $(\alpha)$ must select another (possibly the same) point $x_{2} \in U_{1}$ and in turn player $(\beta)$ must again respond to this by choosing (possibly the same) $U_{2} \in \mathcal{U}(x)$. The players repeat this procedure infinitely many times. We shall say that the player $(\beta)$ wins the $\mathcal{G}(x)$-game if the sequence $\left\langle x_{n}: n \in \mathrm{N}\right\rangle$ has a cluster point in $X$. Otherwise, the player $(\alpha)$ is said to have won the game. By a strategy $\sigma$ for the player $(\beta)$, we mean a 'rule' that specifies each move of player $(\beta)$ in every possible situation. More precisely, a strategy $\sigma=\left\langle\sigma_{n}: n \in \mathbf{N}\right\rangle$ for $(\beta)$ is a sequence of $\mathcal{U}(x)$-valued functions. We shall call a finite sequence $\left\langle x_{1}, x_{2}, \ldots, x_{n}\right\rangle$ or an infinite sequence $\left\langle x_{1}, x_{2}, \ldots\right\rangle$ a $\sigma$-sequence if $x_{i+1} \in \sigma_{i}\left(\left\langle x_{1}, x_{2}, \ldots, x_{i}\right\rangle\right)$ for each $i$ such that $1 \leqslant i<n$ or $x_{n+1} \in \sigma_{n}\left(\left\langle x_{1}, x_{2}, \ldots, x_{n}\right\rangle\right)$ for each $n \in \mathbf{N}$. A strategy $\sigma=\left\langle\sigma_{n}: n \in \mathbf{N}\right\rangle$ for player $(\beta)$ is called $a$ winning strategy if each infinite $\sigma$-sequence has a cluster point in $X$. Finally, we call $x$ a $\mathcal{G}$-point if the player $(\beta)$ has a winning strategy for the $\mathcal{G}(x)$-game. In addition, if every point of $X$ is a $\mathcal{G}$-point, then $X$ is called a $\mathcal{G}$-space.

The class of $\mathcal{G}$-spaces is quite large. In fact, it contains all $q$-spaces $[8]$ and all $W$ spaces [7], thus contains all first countable spaces and all locally compact spaces. In [2], the following question was asked.

Question 2.1. [2] For which spaces $X$ is the space $C_{p}(X)$ isocompact?

Now we answer Question 2.1 in the class of $\mathcal{G}$-spaces.

Theorem 2.2. Let $X$ be a Tychonoff $\mathcal{G}$-space. Then $C_{p}(X)$ is isocompact.

Proof: Let $Y \subseteq C_{p}(X)$ be a closed countably compact subspace. By countable compactness, for every point $x$ in $X$ there is an $M_{x}>0$ such that $|f(x)| \leqslant M_{x}$ for all $f \in Y$. It follows that

$$
Y \subseteq \prod_{x \in X}\left[-M_{x}, M_{x}\right]
$$

Therefore, $\bar{Y}$ is a compact subset of $\mathbf{R}^{X}$, where the closure is taken in $\mathbf{R}^{X}$. We first show that $\bar{Y} \subseteq C_{p}(X)$. To this end, assume that there exists some $g \in \bar{Y} \backslash C_{p}(X)$. Since $g$ is not continuous, there must be some point $x_{0}$ and $\varepsilon>0$ such that for each $U \in \mathcal{U}\left(x_{0}\right)$ we can choose at least one point $x_{U} \in U$ satisfying

$$
\left|g\left(x_{U}\right)-g\left(x_{0}\right)\right| \geqslant \varepsilon
$$

Let $\sigma$ be a winning strategy for player $(\beta)$ in the $\mathcal{G}\left(x_{0}\right)$-game. Without loss of generality, let $x_{0}$ be the first move of player $(\alpha)$. Then player $(\beta)$ responds by $\sigma\left(\left\langle x_{0}\right\rangle\right)$ and $f_{1} \in$ $W\left(x_{0} ; 1\right)(g) \cap Y$. To respond to this, player $(\alpha)$ chooses a point

$$
x_{1} \in \sigma\left(\left\langle x_{0}\right)\right) \cap\left\{x \in X:\left|f_{1}(x)-f_{1}\left(x_{0}\right)\right|<1\right\}
$$


such that

$$
\left|g\left(x_{1}\right)-g\left(x_{0}\right)\right| \geqslant \varepsilon .
$$

Inductively, players $(\alpha)$ and $(\beta)$ produce a $\sigma$-sequence $\left\langle x_{n}: n \in \mathbf{N}\right\rangle$ in $X$ and a sequence $\left\langle f_{n}: n \in \mathrm{N}\right\rangle$ in $Y$ such that

(1) $x_{n} \in \sigma\left(\left\langle x_{0}, x_{1}, \ldots, x_{n-1}\right\rangle\right)$ for each $n \in \mathbf{N}$;

(2) $x_{n} \in \bigcap_{i=1}^{n}\left\{x \in X:\left|f_{i}(x)-f_{i}\left(x_{0}\right)\right|<1 / n\right\}$ for each $n \in \mathbf{N}$;

(3) $\left|g\left(x_{n}\right)-g\left(x_{0}\right)\right| \geqslant \varepsilon$ for each $n \in \mathbf{N}$; and

(4) $f_{n} \in W\left(x_{0}, x_{1}, \ldots, x_{n-1} ; 1 / n\right)(g)$ for each $n \in \mathbf{N}$.

Since $Y$ is closed countably compact, $\left\langle f_{n}: n \in \mathbf{N}\right\rangle$ has a cluster point, say $f$ in $Y$. Let $x_{\infty}$ be a cluster point of the $\sigma$-sequence $\left\langle x_{n}: n \in \mathrm{N}\right\rangle$ in $X$. First of all, $f$ and $g$ coincide on $\left\{x_{n}: n \in \omega\right\}$. To see this, for each fixed $m \in \omega$ and an arbitrary $\delta>0$ we have the following

(5) $\left|f\left(x_{m}\right)-g\left(x_{m}\right)\right| \leqslant\left|f\left(x_{m}\right)-f_{n}\left(x_{m}\right)\right|+\left|f_{n}\left(x_{m}\right)-g\left(x_{m}\right)\right|<\delta$.

whenever $n \in \omega$ is large enough. In particular, $f\left(x_{0}\right)=g\left(x_{0}\right)$. On the other hand, (2) implies that

$$
\left|f_{i}\left(x_{\infty}\right)-f_{i}\left(x_{0}\right)\right|<1 / n
$$

for all $i<n$. Let $n \rightarrow \infty$ and $i \rightarrow \infty$, we have $f\left(x_{\infty}\right)=f\left(x_{0}\right)=g\left(x_{0}\right)$. This contradicts (3). Therefore $g$ must be continuous. We have shown that $\bar{Y} \subseteq C_{p}(X)$.

Therefore, $\bar{Y}=Y$. We have already observed that $\bar{Y}$ is compact, so that $Y$ is compact. The proof is completed.

\section{REFERENCES}

[1] A. Arkhangel'skii, Topological function spaces, Mathematics and its Applications (Kluwer Academic Publishers, Dordrecht, 1992).

[2] A.V. Arkhangel'skii, 'On a theorem of Grothendieck in $C_{p}$-theory', Topology Appl. 80 (1997), 21-41.

[3] P. Bacon, 'The compactness of countably compact spaces', Pacific J. Math. 32 (1970), 587-592.

[4] A. Bouziad, 'The Ellis theorem and continuity in groups', Topology Appl. 50 (1993), 73-80.

[5] J. Chaber, 'Conditions which imply compactness in countable compact spaces', Bull. Acad. Polon. Sci. Sér. Sci Math. Astronom. Phys. 24 (1976), 993-998.

[6] R. Engelking, General topology (Polish Scientific Publisher, Warszawa, 1977).

[7] G. Gruenhage, 'Infinite games and generalizations of first-countable spaces', General Topology and Appl. 6 (1976), 339-352.

[8] E. Michael, 'A note on closed maps and compact sets', Israel J. Math. 2 (1964), 173-176.

[9] S. Nedev, 'Symmetrizable spaces and final compactness', Soviet Math. Dokl. 8 (1967), 890-892. 
[10] J. Worrell, Jr and H. Wicke, 'Characterizations of developable topological spaces', Canad. J. Math. 17 (1965), 820-830.

Department of Mathematics

The University of Auckland

Private Bag 92019

Auckland

New Zealand 